\title{
Estimation of multiple biomass growth rates and biomass concentration in a class of bioprocesses
}

\author{
V. Lubenova, I. Rocha, E.C. Ferreira
}

Abstract In this paper, an approach to the estimation of $C_{i}$ multiple biomass growth rates and biomass concentration is proposed for a class of aerobic bioprocesses characterized by on-line measurements of dissolved oxygen and carbon dioxide concentrations, as well as off-line measurements of biomass concentration. The approach is based on adaptive observer theory and includes two steps. In the first step, an adaptive estimator of two out of three biomass growth rates is designed. In the second step, the third biomass growth rate and the biomass concentration are estimated, using two different adaptive estimators. One of them is based on on-line measurements of dissolved oxygen concentration and off-line measurement of biomass concentrations, while the other needs only on-line measurements of the carbon dioxide concentration. Simulations demonstrated good performance of the proposed estimators under continuous and batch-fed conditions.

Keywords Adaptive observer, Bioprocesses, On-line estimation, Software sensors

List of sym
$\mathbf{A}_{1}, \mathbf{A}_{2}$
$\mathbf{B}_{1 i}, \mathbf{B}_{2 i}$
$C$
$C$
$C^{*}$

List of symbols

$A_{1}, A_{2}$

matrices of the error system

matrices connected with error system, $i=1,2$

carbon dioxide concentration, $\mathrm{g} / \mathrm{l}$ carbon dioxide saturation concentration, $\mathrm{g} / \mathrm{l}$

Received: 6 December 2002 / Accepted: 4 April 2003

Published online: 18 June 2003

(C) Springer-Verlag 2003

V. Lubenova $(\varangle)$

Institute of Control and System Research, Bulgarian Academy of Sciences,

Acad. G. Bonchev str., PO Box 79, 1113 Sofia, Bulgaria

E-mail: lyubenova@icsr.bas.bg

Tel.: +003-59-2732614

Fax: +003-59-28703361

I. Rocha, E.C. Ferreira

Centro de Engenharia Biológica,

Universidade do Minho, 4710-057 Braga, Portugal

Fundação para a Ciência e a Tecnologia (Portugal) provided financial support for I. Rocha through a doctoral research grant (PRAXIS XXI/BD/16961/98) and to V. Lubenova through a post-doctoral research grant (POCTI BPD/6950/2001). Also, V. Lubenova was a recipient of a NATO Science Advanced Fellowship from ICCTI (Portugal).
$C_{x 1}, C_{x 2}$

$C^{\mathrm{I}}, C^{\mathrm{II}}, C^{\mathrm{III}}, C^{\mathrm{IV}}$

CTR

$c_{1}, c_{2}$

$D$

$\mathbf{F}$

$h_{i}^{\mathrm{I}}, h_{i}^{\mathrm{II}}$

$h_{i}^{\mathrm{III}}$

$\mathrm{I}_{2}$

$\mathrm{K}_{\mathrm{si}}$

$K_{i}$

$k_{i}$

$k l a_{\mathrm{o}}, k l a_{\mathrm{c}}$

$1_{0 i}, 1_{1 i}, l_{2 i}$

$M_{i}$

$O$

$O^{*}$

OTR

$\mathbf{P}_{1}, \mathbf{P}_{2}$

$P_{2}$

Q

$\mathbf{r}(\xi)$

$R_{1}, R_{2}, R_{3}$

$\hat{R}_{2 t}$

$S_{1}, S_{2}$

$S_{\text {in } 1}$

$T_{x}$

$\mathbf{u}_{1}, \mathbf{u}_{2}$

$V_{1}, V_{2}$

$X$

$\mathrm{x}_{1}, \mathrm{x}_{2}$ estimator parameters, $i=1-10$

design parameters of estimator II

design parameters of estimators I and II

carbon dioxide transfer rate, $\mathrm{g} / \mathrm{l} / \mathrm{min}$ auxiliary parameters

dilution rate/min

feed rate vector with $\operatorname{dim}(F)=n$ eigenvalues of the matrices $B_{11}, B_{21}$, respectively, $i=1,2$

eigenvalues, related to the error system of estimator III, $i=1,2$ identity matrix with $\operatorname{dim}\left(\mathbf{I}_{2}\right)=2$ saturation constants, $i=1,2,3 \mathrm{~g} / 1$ inhibition constant, $\mathrm{g} / \mathrm{l}$ yield coefficients, $i=1-9$ $n \times m$ yield coefficient matrix mass transfer coefficients for dissolved oxygen and dioxide carbon concentrations/min positive constants, $i=1,2$ upper bounds, $i=1-4$ dissolved oxygen concentration, $\mathrm{g} / \mathrm{l}$ dissolved oxygen saturation concentration, $\mathrm{g} / \mathrm{l}$ oxygen transfer rate, $\mathrm{g} / \mathrm{l} / \mathrm{min}$ matrices, connected with error system of estimators I and II product $\mathrm{P}_{2}$ concentration, $\mathrm{g} / \mathrm{l}$ the gaseous outflow rate vector with $\operatorname{dim}(Q)=n$ reaction rate vector, $\operatorname{dim}(r)=m$ biomass growth rates, related to respiratory growth on substrate $S_{1}$, to fermentative growth on substrate $S_{1}$, and to respiratory growth on substrate $S_{2}$ $(P)$, respectively, $\mathrm{g} / \mathrm{l} / \mathrm{min}$ estimates of $R_{2}$ using adaptive estimator III, $\mathrm{g} / \mathrm{l} / \mathrm{min}$ substrate $S_{1}$ and $S_{2}$ concentrations, g/l concentration of the substrate $S_{1}$ in the feed, $\mathrm{g} / \mathrm{l}$

sampling time of the off-line biomass measurements, min input vectors of the error systems auxiliary variables of estimators I and II respectively biomass concentration, $\mathrm{g} / \mathrm{l}$ estimation error vectors 


\begin{tabular}{|c|c|}
\hline Greek & \\
\hline$\beta_{1 i}, \beta_{2 i}, \delta_{1}, \delta_{2}, \gamma_{1}, \gamma_{2}$ & positive constants, $i=1,2$ \\
\hline$\varrho_{1}, \varrho_{2}, \varrho_{3}$ & $\begin{array}{l}\text { auxiliary parameters of the esti- } \\
\text { mators I and II }\end{array}$ \\
\hline$\xi$ & $\begin{array}{l}\text { vector of state variables with } \\
\operatorname{dim}(\xi)=n\end{array}$ \\
\hline$\mu_{1}, \mu_{2}, \mu_{3}$ & $\begin{array}{l}\text { specific growth rates, connecting to } \\
R_{1}, R_{2}, R_{3} \text {, respectively, } \mathrm{min}^{-1}\end{array}$ \\
\hline
\end{tabular}

\section{Superscripts}

$\wedge$

$\sim$

\section{Subscripts}

$\max i$

$$
\text { maximum values }(i=1-3)
$$

process. In both cases, two partial models were used to describe the process.

One of the problems connected with the monitoring and control of bioprocesses is the absence of frequent (online) measurements of some essential variables. In these cases, continuous estimates of these variables can be obtained from available frequent and/or infrequent (off-line) measurements, using a model of the process under consideration as well as the above-mentioned estimation algorithms. In recent years, the problem of multi-rate state and parameter estimation has received more attention, with emphasis on the extension of the EKF for processes with multi-rate/delayed measurements $[15,16,17,18,19$, 20]. Multi-rate variables estimation allows for using infrequently available measurements in state and parameter estimation, leading to a considerable improvement in the accuracy of the resulting estimates. The inadequacy of the EKF for several nonlinear processes, as reported by Gudi [15], and the recent advances in nonlinear state estimator design, motivate the use of nonlinear multi-rate state estimators that can effectively handle process nonlinearities and multi-rate measurements $[1,21]$.

This paper is dedicated to the estimation of multiple biomass growth rates and biomass concentration of a class of bioprocesses, characterized by the following reaction network:

$S_{1}+O \stackrel{\mu_{1}}{\longrightarrow} X+C$

$S_{1} \stackrel{\mu_{2}}{\longrightarrow} X+C+S_{2}(P)$

$S_{2}(P)+O \stackrel{\mu_{3}}{\longrightarrow} X+C$

where $S_{1}$ is the first substrate, $O$ is dissolved oxygen, $X$ is biomass, $S_{2}(P)$ is the second substrate, which can be a product depending on the metabolic pathway, $C$ is carbon dioxide, and $\mu_{1}, \mu_{2}$, and $\mu_{3}$ are the specific growth rates for the three metabolic pathways. In the following, $O, X, S_{1}, S_{2}$, and $C$ will denote the concentrations of these variables.

Pathways (1), (2) and (3) refer respectively to the respiratory growth on substrate $S_{1}$ (oxidative pathway), the fermentative growth on substrate $S_{1}$ (reductive pathway), and the respiratory growth on $S_{2}(P)$ (oxidative pathway). For example, the above reaction network can describe baker's yeast batch-fed fermentation [22], where $S_{1}$ is the glucose concentration, $S_{2}(P)$ is the ethanol concentration. Some processes of the production of recombinant proteins with E. coli $[23,24,25]$ can also be described using the above scheme, using a switch between two partial sub-models. In this case, $S_{1}$ is again the glucose concentration and $S_{2}(P)$ denotes the acetate concentration.

This paper considers the case when the processes, belonging to the class above, are described using only one full model instead of partial sub-models. Three adaptive estimation algorithms are proposed. The first estimates two of the biomass growth rates $R_{1}$ and $R_{3}$, using on-line measurements of the dissolved oxygen concentration. The second algorithm estimates the biomass growth rate $R_{2}$ and biomass concentration, using additionally $X$ off-line measurements. The third estimator is observer-based and estimates $R_{2}$ using on-line 
measurements of the carbon dioxide concentration. The second and third estimators need estimates $R_{1}$ and $R_{3}$, obtained by the first algorithm. The estimator design is based on adaptive estimation theory. The performances of the proposed estimators are investigated and compared by simulations for various kinds of processes.

\section{2}

\section{Statement of problem}

A general dynamical model of a stirred tank reactor [2] has the form:

$\frac{\mathrm{d} \xi}{\mathrm{d} t}=\mathbf{K r}(\xi)-\mathrm{D} \xi+\mathbf{F}-\mathbf{Q}$

where $\xi$ is the vector of state variables with $\operatorname{dim}(\xi)=n, \mathrm{~K}$ is the $n \times m$ yield coefficient matrix, $D$ is the dilution rate, $\boldsymbol{F}$ is the feed rate vector with $\operatorname{dim}(\boldsymbol{F})=n, \boldsymbol{Q}$ is the gaseous outflow rate vector with $\operatorname{dim}(\boldsymbol{Q})=n, \boldsymbol{r}(\xi)$ is a reaction rate vector with $\operatorname{dim}(\boldsymbol{r})=m$.

The dynamics of this class of bioprocesses, which is characterized by reaction schemes (1), (2), and (3), is given in the matrix form (4), by the following vectors and matrix:

$\xi=\left[\begin{array}{c}X \\ S_{1} \\ S_{2}(P) \\ O \\ C\end{array}\right] ; \mathbf{K}=\left[\begin{array}{ccc}1 & 1 & 1 \\ -k_{1} & -k_{2} & 0 \\ 0 & k_{3} & -k_{4} \\ -k_{5} & 0 & -k_{6} \\ k_{7} & k_{8} & k_{9}\end{array}\right] ;$
$\mathbf{F}=\left[\begin{array}{c}0 \\ D S_{\text {in } 1} \\ 0 \\ \text { OTR } \\ 0\end{array}\right] ; \mathbf{Q}=\left[\begin{array}{c}0 \\ 0 \\ 0 \\ 0 \\ C T R\end{array}\right] ; \mathbf{r}(\xi)=\left[\begin{array}{l}\mu_{1} X \\ \mu_{2} X \\ \mu_{3} X\end{array}\right]=\left[\begin{array}{l}R_{1} \\ R_{2} \\ R_{3}\end{array}\right]$

where $k_{1}-k_{9}$ are yield coefficients, OTR is the oxygen transfer rate, CTR is the carbon dioxide transfer rate, and $S_{\text {in } 1}$ is the first substrate concentration in the feed.

For system (4), (5), it is assumed that:

- A1. The measurements of $X, S_{1}$ and $S_{2}(P)$ are not available on-line, while $O, C$, oxygen transfer rate, $O T R$, and the carbon dioxide transfer rate, $C T R$, are measured on-line.

- A2. The elements of the yield coefficient matrix $\mathrm{K}$ are known and constants.

- A3. The off-line measurements of biomass concentration are available.

- A4. The biomass growth rates, $R_{1}, R_{2}$, and $R_{3}$, are considered to be unknown, time-varying parameters.

For the class of bioprocesses, described by system (4), (5) and under assumptions A1-A4, the following problem is considered: estimation of the biomass growth rates $R_{1}$, $R_{2}$, and $R_{3}$, as well as biomass concentration $X$, by algorithms derived by the theory of adaptive estimation using on-line measurements of the dissolved oxygen, carbon dioxide concentration and the off-line measurements of the biomass.

\section{Adaptive algorithm design}

On the basis of the available a priori information, formulated in assumptions A1-A4 and process model (4), it is easy to establish that the structure of an observerbased estimator of the reaction rates, $R_{1}, R_{2}$, and $R_{3}$, including the dynamical equations of dissolved oxygen, carbon dioxide concentrations, and the reaction rates, is not observable. For this reason, in this paper, a two-step estimation approach is proposed. In the first step, an adaptive estimator of $R_{1}$ and $R_{3}$ (estimator $\mathrm{I}$ ) is derived on the basis of measurements of dissolved oxygen concentration. The biomass concentration and the biomass growth rate $R_{2}$ are estimated in the second step, using different adaptive estimators (estimator II and estimator III). The first is based on on-line measurements of dissolved oxygen concentration and off-line measurements of biomass concentration, while the other needs on-line measurements of carbon dioxide concentration. Both of the estimators (II and III) use the estimates of $R_{1}$ and $R_{3}$, obtained in the first step.

\section{4}

\section{Estimator I - estimation of $\boldsymbol{R}_{\mathbf{1}}$ and $\boldsymbol{R}_{\mathbf{3}}$}

According to model (4), (5), the dynamics of dissolved oxygen concentration, $O$, are given by the following equation:

$\frac{\mathrm{d} O}{\mathrm{~d} t}=-k_{5} R_{1}-k_{6} R_{3}-D O+O T R$

From Eq. (6), it follows that the oxygen uptake rate $(O U R)$ of the micro-organisms is given by:

$O U R=k_{5} R_{1}+k_{6} R_{3}$

Then the time derivative of reaction rate $R_{1}$ can be presented as follows:

$\frac{\mathrm{d} R_{1}}{\mathrm{~d} t}=\frac{\mathrm{d} O U R}{\mathrm{~d} t} \frac{1}{k_{5}}-k_{6} \frac{\mathrm{d} R_{3}}{\mathrm{~d} t} \frac{1}{k_{5}}$

Define the auxiliary parameter:

$\rho_{1}=\left(\frac{\mathrm{d} O U R}{\mathrm{~d} t} \frac{1}{k_{5}}-k_{6} \frac{\mathrm{d} R_{3}}{\mathrm{~d} t} \frac{1}{k_{5}}+C^{\mathrm{I}} R_{1}\right) \frac{1}{C^{\mathrm{II}}}$

with $C^{\mathrm{I}}$ and $C^{\mathrm{II}}$ positive constants or time-varying

parameters.

By combining Eqs. (6), (8), and (9), it is possible to derive the following adaptive estimator of $R_{1}$ and $R_{3}$ :

$\frac{\mathrm{d} \hat{O}}{\mathrm{~d} t}=-k_{5} \hat{R}_{1}-k_{6} \hat{R}_{3}-D O+O T R+C_{1}(O-\hat{O})$

$\frac{\mathrm{d} \hat{R}_{1}}{\mathrm{~d} t}=C^{\mathrm{II}} \hat{\rho}_{1}-C^{\mathrm{I}} \hat{R}_{1}+C_{2}(O-\hat{O})-C_{4} V_{1}(O-\hat{O})$

$\frac{\mathrm{d} \hat{R}_{3}}{\mathrm{~d} t}==-C_{3}(O-\hat{O})$

$\frac{\mathrm{d} \hat{\rho}_{1}}{\mathrm{~d} t}=-C_{4}(O-\hat{O})$ 
$\frac{\mathrm{d} V_{1}}{\mathrm{~d} t}=-C^{\mathrm{I}} V_{1}+C^{\mathrm{II}}$

where $\hat{O}, \hat{R}_{1}, \hat{R}_{3}$ and $\hat{\rho}_{1}$ are estimates for $O, R_{1}, R_{3}$ and $\varrho_{1}$, $C_{1}, C_{2}, C_{3}$ and $C_{4}$ are estimator parameters, which must be chosen according to stability conditions, and $V_{1}$ is an auxiliary variable. The dynamical equations of $\hat{R}_{3}$ (12) and $\hat{\rho}_{1}(13)$ are driven by the error $(O-\hat{O})$, which is supposed to reflect the deviation between $\hat{R}_{3}$ and $R_{3}\left(\varrho_{1}\right.$ and $\hat{\rho}_{1}$ respectively).

The introduction of the auxiliary parameters $\varrho_{1}, C^{\mathrm{I}}$,

$C^{\mathrm{II}}$, and auxiliary variable $V_{1}$ is a necessary condition for the observer design to be achieved using theoretical results concerning the stability of adaptive observers, as shown in the following section.

\section{5}

\section{Stability analysis}

Defining the estimation errors:

$$
\begin{aligned}
& \tilde{O}=O-\hat{O} ; \quad \tilde{R}_{1}=R_{1}-\hat{R}_{1} ; \quad \tilde{R}_{3}=R_{3}-\hat{R}_{3} ; \\
& \tilde{\rho}_{1}=\rho_{1}-\hat{\rho}_{1}
\end{aligned}
$$

the following error system is obtained:

$\frac{\mathrm{d} \mathbf{x}_{1}}{\mathrm{~d} t}=\mathbf{A}_{1} \mathbf{x}_{1}+\mathbf{u}_{1}$

where

$$
\begin{aligned}
\mathbf{x}_{1} & =\left[\begin{array}{c}
\tilde{O} \\
\tilde{R}_{1} \\
\tilde{R}_{3} \\
\tilde{\rho}_{1}
\end{array}\right] ; \quad \mathbf{A}_{1}=\left[\begin{array}{cccc}
-C_{1} & -k_{5} & -k_{6} & 0 \\
-C_{2}+C_{4} V_{1} & -C^{\mathrm{I}} & 0 & C^{\mathrm{II}} \\
C_{3} & 0 & 0 & 0 \\
C_{4} & 0 & 0 & 0
\end{array}\right] ; \\
\mathbf{u}_{1} & =\left[\begin{array}{ll}
0 & 0 \\
0 & 0 \\
1 & 0 \\
0 & 1
\end{array}\right]\left[\begin{array}{c}
\frac{\mathrm{d} R_{3}}{\mathrm{~d} t} \\
\frac{\mathrm{d} \rho_{1}}{\mathrm{~d} t}
\end{array}\right]
\end{aligned}
$$

It is assumed that:

- A5. $C_{1}>0 ; C_{1}=h_{1}+h_{2}-\mathrm{C}^{\mathrm{I}}$

- A6. $C_{2}<0 ; C_{2}=\frac{\left(h_{2}-h_{1}\right)^{2}-\left(C_{1}-C^{\mathrm{I}}\right)^{2}}{4 k_{5}}$.

- A7. $c_{1}>0 ; c_{1}=-C_{2} / k_{5}$.

- A8. $C_{3=} \mathrm{c}_{1} \mathrm{k}_{6} ; C_{4=} \mathrm{c}_{1} \mathrm{k}_{5} \mathrm{~V}_{1}$, where $h_{1}=-h_{1}{ }^{\prime}, h_{2}=-h_{2}{ }^{\prime}$ with $h_{1}$, $h_{2}$ positive constants, and $h_{1}{ }^{\prime}, h_{2}{ }^{\prime}$ eigenvalues of the matrix:

$$
\mathbf{B}_{11}=\left[\begin{array}{ll}
-C_{1} & -k_{5} \\
-C_{2} & -C^{\mathrm{I}}
\end{array}\right]
$$

- A9. Vector $\left[k_{6}, \mathrm{k}_{5} \mathrm{~V}_{1}\right]^{T}$ is a persistently exciting signal, i.e., there exist positive constants $\delta_{1}, \beta_{11}, \beta_{21}$, such that, for all $t$

$$
0<\beta_{11} \mathbf{I}_{2} \leq \int_{t}^{t+\delta_{1}}\left[\begin{array}{c}
k_{6} \\
k_{5} V_{1}
\end{array}\right]\left[\begin{array}{ll}
k_{6} & k_{5} V_{1}
\end{array}\right] \mathrm{d} \delta_{1} \leq \beta_{21} \mathbf{I}_{2}<\infty
$$

where $I_{2}$ is the identity matrix.

- A10. $\left|\frac{\mathrm{d} R_{3}}{\mathrm{~d} t}\right| \leq M_{1}$

- A11. $\left|\frac{\mathrm{d} \rho_{1}}{\mathrm{~d} t}\right| \leq M_{2}$ where $M_{1}$ and $M_{2}$ are upper bounds.

Statement 1 Under assumptions A1-A11, there exist positive finite constants $l_{01}, l_{11}$, and $l_{21}$ such that the error $x_{1}$ is bounded as follows, for all $t$

$\left\|\mathbf{x}_{1}(t)\right\| \leq l_{01}\left\|\mathbf{x}_{1}(0)\right\|+l_{11} M_{1}+l_{21}$

The proof is given in the Appendix.

\section{6}

\section{Estimator II - estimation of $R_{2}$ and $X$}

Define the auxiliary parameters:

$\rho_{2}=O U R+X$

$\rho_{3}=\left(-R_{2}-C^{\mathrm{III}} X\right) \frac{1}{C^{\mathrm{IV}}}$

where $C^{\mathrm{III}}$ and $C^{\mathrm{IV}}$ can be positive constants or timevarying parameters.

The dynamics of dissolved oxygen concentration, given by Eq. (6) and using Eq. (16) can be presented as follows:

$\frac{\mathrm{d} O}{\mathrm{~d} t}=-\rho_{2}+X-D O+O T R$

According to Eq. (17), the dynamical model of $X$ can be written as:

$\frac{\mathrm{d} X}{\mathrm{~d} t}=R_{1}+R_{3}-C^{\mathrm{IV}} \rho_{3}-C^{\mathrm{III}} X-D X$

Using Eqs. (18), (19), the following adaptive estimator can be derived:

$\frac{\mathrm{d} \hat{O}}{\mathrm{~d} t}=-\hat{\rho}_{2}+\hat{X}-D O+O T R+C_{5}(O-\hat{O})$

$\begin{aligned} \frac{\mathrm{d} \hat{X}}{\mathrm{~d} t}= & \hat{R}_{1}+\hat{R}_{3}-C^{\mathrm{IV}} \hat{\rho}_{3}-C^{\mathrm{III}} \hat{X}-D \hat{X}+C_{6}(O-\hat{O}) \\ & +C_{8} V_{2}(O-\hat{O})+C_{\mathrm{x}}\left(X_{\mathrm{L}}-\hat{X}_{\mathrm{L}}\right)\end{aligned}$

$\frac{\mathrm{d} \hat{\rho}_{2}}{\mathrm{~d} t}=-C_{7}(O-\hat{O})$

$\frac{\mathrm{d} \hat{\rho}_{3}}{\mathrm{~d} t}=-C_{8}(O-\hat{O})-C_{x 2}\left(X_{2}-\hat{X}_{2}\right)$

$\frac{\mathrm{d} V_{2}}{\mathrm{~d} t}=-\left(C^{\mathrm{III}}+D\right) V_{2}+C^{\mathrm{IV}}$

where $\hat{O}, \hat{X}, \hat{\rho}_{2}$, and $\hat{\rho}_{3}$ are estimated values for $O, X, \varrho_{2}$ and $\varrho_{3}, C^{\mathrm{III}}, C^{\mathrm{IV}}$ are auxiliary parameters, $C_{5}-C_{8}, C_{x 1}$, and $C_{x 2}$ are estimator parameters, $V_{2}$ is an auxiliary variable, $X_{\mathrm{L}}$ and $\hat{X}_{\mathrm{L}}$ are the off-line measurements and the estimates of $X$, and $\hat{R}_{1}, \hat{R}_{3}$ are the estimated values of the biomass growth rates $R_{1} R 2$ obtained by the first algorithm.

An estimation of $R_{2}$ can be obtained using the estimates of $\varrho_{3}, X$, and the relationship (17) in the following way:

$\hat{R}_{2}=-\hat{\rho}_{3} C^{\mathrm{IV}}-C^{\mathrm{III}} \hat{X}$ 
Here, similarly to estimator I, the introduction of the auxiliary parameters $\varrho_{2}, \varrho_{3}, C^{\mathrm{III}}, C^{\mathrm{IV}}$, and auxiliary variable $V_{2}$ must be considered as a necessary condition of the estimator design.

\section{1}

\section{Stability analysis of estimator II without off-line measurements}

Defining the estimation errors

$$
\tilde{O}=O-\hat{O} \quad \tilde{X}=X-\hat{X} \quad \tilde{\rho}_{2}=\rho_{2}-\hat{\rho}_{2} \quad \tilde{\rho}_{3}=\rho_{3}-\hat{\rho}_{3}
$$

the following error system is obtained:

$\frac{\mathrm{d} x_{2}}{\mathrm{~d} t}=\mathbf{A}_{2} x_{2}+u_{2}$

where

$$
\begin{aligned}
x_{2} & =\left[\begin{array}{c}
\tilde{O} \\
\tilde{X} \\
\tilde{\rho}_{2} \\
\tilde{\rho}_{3}
\end{array}\right], \\
\mathbf{A}_{2} & =\left[\begin{array}{cccc}
-C_{5} & 1 & -1 & 0 \\
-C_{6}-C_{8} V_{2} & -C^{\mathrm{III}}-D & 0 & -C^{\mathrm{IV}} \\
C_{7} & 0 & 0 & 0 \\
C_{8} & 0 & 0 & 0
\end{array}\right], \\
u_{2} & =\left[\begin{array}{ll}
0 & 0 \\
0 & 0 \\
1 & 0 \\
0 & 1
\end{array}\right]\left[\begin{array}{l}
\mathrm{d} \rho_{2} \\
\mathrm{~d} t \\
\frac{\mathrm{d} \rho_{3}}{\mathrm{~d} t}
\end{array}\right]
\end{aligned}
$$

It is assumed that:

- A12. $C_{5}>0 ; C_{5}=2 h-\left(C^{\mathrm{III}}+D\right)$ with $h_{1}{ }^{\mathrm{II}}=h_{2}{ }^{\mathrm{II}}=-h, h$ is a positive constant, $h_{1}{ }^{\mathrm{II}}, h_{2}{ }^{\mathrm{II}}$ are eigenvalues of the matrix:

$\mathbf{B}_{21}=\left[\begin{array}{cc}-C_{5} & 1 \\ -C_{6} & -C^{\mathrm{III}}-D\end{array}\right]$

- A13. $C_{6}>0 ; C_{6}=\frac{\left[C_{5}-\left(C^{\mathrm{III}}+D\right)\right]^{2}}{4}$

- A14. $c_{2}>0 ; c_{2}=C_{6}$

- A15. $C_{7=} \mathrm{c}_{2} ; C_{8}=\mathrm{c}_{2} \mathrm{~V}_{2}$

- A16. Vector $\left[1, V_{2}\right]^{T}$ is a persistently exciting signal, i.e., there exist positive constants $\delta_{2}, \beta_{12}$, and $\beta_{22}$ such that, for all $t$

$0<\beta_{12} \mathbf{I}_{2} \leq \int_{t}^{t+\delta_{2}}\left[\begin{array}{c}1 \\ V_{2}\end{array}\right]\left[\begin{array}{ll}1 & V_{2}\end{array}\right] \mathrm{d} \delta_{2} \leq \beta_{22} \mathbf{I}_{2}<\infty$

- A17. $\left|\frac{\mathrm{d} \rho_{2}}{\mathrm{~d} t}\right| \leq M_{3}$

- A18. $\left|\frac{\mathrm{d} \rho_{3}}{\mathrm{~d} t}\right| \leq M_{4}$, where $M_{3}$ and $M_{4}$ are upper bounds.

Statement 2 Under assumptions A11-A18, there exist positive finite constants $l_{02}, l_{12}$, and $l_{22}$, such that the error $\mathbf{x}_{2}$ is bounded as follows, for all $t$

$\left\|\mathbf{x}_{2}(t)\right\| \leq l_{02}\left\|\mathbf{x}_{2}(0)\right\|+l_{12} M_{4}+l_{22}$

The proof is given in the Appendix.
6.2

\section{Stability analysis of estimator II with off-line measurements}

When off-line measurements of the biomass concentration are available, it is assumed that:

- A19. $C_{5}>0 ; C_{5}=2 h-\left(C^{\mathrm{III}}+C_{x 1}+D\right)$, with $h_{1}{ }^{\mathrm{II}}=h_{2}{ }^{\mathrm{II}}=-\mathrm{h}, h$ is a positive constant, $h_{1}$ II,$h_{2}$ II are eigenvalues of the matrix:

$$
\mathbf{B}_{21}=\left[\begin{array}{cc}
-C_{5} & 1 \\
-C_{6} & -C^{\mathrm{III}}-C_{x 1}-D
\end{array}\right]
$$

- A20. $C_{6}>0 ; C_{6}=\left\{C_{5}-\left(C^{\mathrm{III}}+C_{x 1}+D\right)\right\}^{2} / 4$.

- A21. $c_{2}>0 ; c_{2}=C_{6}$.

- A22. $C_{7=} \gamma_{1}$, with $\gamma_{1}$ a positive constant.

- A23. $C_{8}=0$.

- A24. $\mathrm{C}_{\mathbf{x} 2=\gamma_{2}} \mathrm{C}^{\mathrm{IV}}$, with $\gamma_{2}$ a positive constant.

Then the dynamics of the estimation errors vector $\boldsymbol{x}_{2}$ will be described by Eq. (26), where

$\mathbf{A}_{2}=\left[\begin{array}{cccc}-C_{5} & 1 & -1 & 0 \\ -C_{6}-C_{8} V_{2} & -C^{\mathrm{III}}-C_{\mathbf{x} 1}-D & 0 & -C^{\mathrm{IV}} \\ C_{7} & 0 & 0 & 0 \\ 0 & C_{\mathrm{x} 2} & 0 & 0\end{array}\right]$

and $\boldsymbol{u}_{2}$ as stated in Eq. (26).

Under assumptions A19-A24, estimator II with off-line measurements stability can be proved in the same way as in statements 1 and 2 .

Estimators I and II include the dynamics of the auxiliary variables $V_{1}$ and $V_{2}$ respectively. Similar approaches for the estimation of other time-varying parameters in a class of bioprocesses, different from the one considered here, have been proposed in [3] and [8]. The dynamical equations of the auxiliary variable $V$ from [3] and [8] are characterized by the lack of design parameter and by the presence of one design parameter respectively. In contrast to these $V$ dynamics, each dynamical equation of $V_{1}$ and $V_{2}$ considered in this paper includes two design parameters: $C^{\mathrm{I}}, \mathrm{C}^{\mathrm{II}}$ and $C^{\mathrm{III}}, C^{\mathrm{N}}$ respectively. As mentioned above, it is a necessary condition for the design of estimators I and II to be realized (Appendix). Another difference, between the estimation algorithms in [3] and [8] and the estimators presented in this paper results from the fact that the estimation in [3] and [8] is obtained using only one estimator, while the state and parameters estimation of the considered class of bioprocesses in this paper requires a two-step approach including two estimators. Their structures are similar to those from the abovementioned papers.

\section{7}

\section{Estimator III - estimation of $R_{2}$ on the basis of on-line measurements of carbon dioxide concentration} In the case where the measurements of carbon dioxide concentration, $C$, are available, the following observerbased estimator can be applied: 


$$
\frac{\mathrm{d} \hat{C}}{\mathrm{~d} t}=-k_{7} \hat{R}_{1}-k_{8} \hat{R}_{2 t}-k_{9} \hat{R}_{3}-D C+C T R+C_{9}(C-\hat{C})
$$

$\frac{\mathrm{d} \hat{R}_{2 t}}{\mathrm{~d} t}=C_{10}(C-\hat{C})$

where $\hat{R}_{2 t}$ and $\hat{C}$ are estimated values of $R_{2 t}$, and $C, C_{9}$, and $C_{10}$ are design parameters.

If the design parameters, $C_{9}$ and $C_{10}$, are chosen as follows:

- A25. $C_{9}=2 h_{1,2}^{\mathrm{III}} C_{10}=0.25 C_{9}^{2} / k_{8}$, where $h_{1,2}{ }^{\mathrm{III}}=h$, with $h_{1,2}$ III eingenvalues, related to the error system, and $h$ is a positive constant, the stability of the estimator III can be proved.

A stable estimate of $X$ can be derived using the following equation:

$\frac{\mathrm{d} \hat{X}}{\mathrm{~d} t}=\hat{R}_{1}+\hat{R}_{3}+\hat{R}_{2 t}-D \hat{X}$

\section{8}

\section{Results and discussion}

The behavior of estimators is investigated by simulating a process model, which belongs to the class defined by Eqs. (4) and (5). The dynamical equations are:

$\frac{\mathrm{d} X}{\mathrm{~d} t}=R_{1}+R_{2}+R_{3}-D X$

$\frac{\mathrm{d} S_{1}}{\mathrm{~d} t}=-k_{1} R_{1}-k_{2} R_{2}-D\left(S_{\text {in }}-S\right)$

$\frac{\mathrm{d} S_{2}}{\mathrm{~d} t}=k_{3} R_{2}-k_{4} R_{3}-D S_{2}$

$\frac{\mathrm{d} O}{\mathrm{~d} t}=-k_{5} R_{1}-k_{6} R_{3}-D O+O T R$

$\frac{\mathrm{d} C}{\mathrm{~d} t}=k_{7} R_{1}+k_{8} R_{2}+k_{9} R_{3}-D C-C T R$

where

$$
\begin{aligned}
& R_{1}=\mu_{1} X, R_{2}=\mu_{2} X, R_{3}=\mu_{3} X ; \quad O T R=k l a_{\mathrm{o}}\left(O^{*}-O\right) \\
& \quad C T R=k l a_{\mathrm{c}}\left(C-C^{*}\right)
\end{aligned}
$$

where $O^{*}$ and $C^{*}$-saturation concentrations of the dissolved oxygen and carbon dioxide respectively, and $k l a_{\mathrm{o}}$, $k l a_{\mathrm{c}}$ mass transfer coefficients for dissolved oxygen and dioxide carbon concentrations.

It is assumed that the specific growth rates $\mu_{1}, \mu_{2}$ only depend on $S_{1}$, while the specific growth rate $\mu_{3}$ depends on $S_{1}$ and $S_{2}$. The kinetic models assumed are:

$$
\begin{aligned}
& \mu_{1}=\mu_{\max 1} S_{1} /\left(K_{\mathrm{s} 1}+S_{1}\right) ; \quad \mu_{2}=\mu_{\max 2} S_{1} /\left(K_{\mathrm{s} 2}+S_{1}\right) ; \\
& \mu_{3}=\mu_{\max 3} S_{2} /\left(K_{\mathrm{s} 3}+S_{2}\right)\left(K_{\mathrm{i}}+S_{1}\right)
\end{aligned}
$$

with constant parameters $\mu_{\max 1}, \mu_{\max 2}, \mu_{\max 3}$, maximum specific growth rates, $K_{\mathrm{s} 1}, K_{\mathrm{s} 2}, K_{\mathrm{s} 3}$ saturation constants, and $K_{\mathrm{i}}$ the inhibition constant. The pseudo-measurements of $X, S_{1}, S_{2}, O$, and $C$ are obtained using the integration of Eqs. (30), (31) (32), (33), and (34). The values of the model parameters used in Eqs. (30), (31) (32), (33), (34), and (35) are given in Table 1.

Two kinds of process operation are considered. For the continuous operation ( $D=$ constant), the initial values of $X$, $S_{1}, S_{2}, O, C, R_{1}, R_{2}, R_{3}$ are given in Table 2 and correspond to a steady-state solution. The initial values of the estimates $\hat{R}_{1}, \hat{R}_{2}, \hat{R}_{3}, \hat{\rho}_{1}, \hat{\rho}_{2}, \hat{\rho}_{3}$ and $\hat{X}$ are chosen to be different from the true values to test the effect of a $10 \%$ error of the initial values of the estimators. The design parameters of estimator I are chosen, using tuning procedure, proposed by assumptions $\mathrm{A}_{5}-\mathrm{A}_{8}$ and $C^{\mathrm{I}}$, a positive constant, $C^{\mathrm{II}}=O$. In Fig. 1, a plot of the estimates $\hat{R}_{1}, \hat{R}_{3}$ and the real values of these parameters are given for different values of $h_{1,2}$. The results show that the changes in this parameter, as well as in $C^{\mathrm{I}}$ and $V_{1}(0)$ (results not shown), lead to changes in the convergence rate of the estimates to their true values. The following values of the parameters $C^{\mathrm{I}}, h_{1,2}{ }^{\mathrm{I}}$, and $V_{1}(0)$ are chosen as "optimal" ones for the process considered and used in the simulation of estimators II and III: $C^{\mathrm{I}}=0.0001$; $h_{1,2} \mathrm{I}=-1$ and $V_{1}(0)=3.5$. The results from estimators II and III are shown in Fig. 2. The design parameters of estimator II are chosen, using the tuning procedure proposed by assumptions $A_{12}-A_{15}$ and $A_{19}-A_{24}$. An improvement in the

\begin{tabular}{|c|c|c|c|c|}
\hline$\mu_{\max 1}=0.01\left(\min ^{-1}\right)$ & $\mu_{\max 2}=0.135\left(\min ^{-1}\right)$ & & $\mu_{\max 3}=0.01\left(\mathrm{~min}^{-1}\right)$ & \\
\hline $\begin{array}{l}K_{\mathrm{i}}=5(\mathrm{~g} / \mathrm{l}) \\
K_{\mathrm{sl}}=5(\mathrm{~g} / \mathrm{l}) \\
K_{1}=0.2 \\
k_{5}=0.05\end{array}$ & $\begin{array}{l}k l a_{\mathrm{o}}=19\left(\mathrm{~min}^{-1}\right) \\
K_{\mathrm{s} 2}=20(\mathrm{~g} / \mathrm{l}) \\
k_{2}=0.1 \\
k_{6}=0.04\end{array}$ & $\begin{array}{l}K_{\mathrm{s} 3}=5(\mathrm{~g} / \mathrm{l}) \\
k_{3}=0.7 \\
k_{7}=0.1\end{array}$ & $\begin{array}{l}k l a_{\mathrm{o}}=17\left(\mathrm{~min}^{-1}\right) \\
O^{*}=0.008(\mathrm{~g} / \mathrm{l}) \\
k_{4}=0.1 \\
k_{8}=0.08\end{array}$ & $\begin{array}{l}C^{*}=0.0053(\mathrm{~g} / \mathrm{l}) \\
\mathrm{S}_{\mathrm{in}}=10(\mathrm{~g} / \mathrm{l}) \\
k_{9}=0.08\end{array}$ \\
\hline
\end{tabular}
estimate convergence is obtained by decreasing the sampling time $T_{x}$ of off-line measurements of $X$. In Fig. 2e, f, $\hat{R}_{2}$ and $\hat{X}$ by estimator II and by estimator III are plotted for

Table 1. Model parameters

Table 2. Initial conditions

\begin{tabular}{llll}
$X(0)=5.13 \mathrm{~g} / \mathrm{l}$ & \multicolumn{2}{c}{$S_{1}(0)=9.42 \mathrm{~g} / \mathrm{l}$} & \multicolumn{2}{c}{$S_{2}(0)=3.1 \mathrm{~g} / \mathrm{l}$} \\
$O(0)=0.0079 \mathrm{~g} / \mathrm{l}$ & $C(0)=0.09 \mathrm{~g} / \mathrm{l}$ & & $R_{1}(0)=0.033 \mathrm{~g} / \mathrm{l} / \mathrm{min}$ \\
$R_{3}(0)=0.00136 \mathrm{~g} / \mathrm{l} / \mathrm{min}$ & & $R_{2}(0)=0.05 / \mathrm{min}$ & $\mathrm{g} / \mathrm{l} / \mathrm{min}$ \\
\hline
\end{tabular}



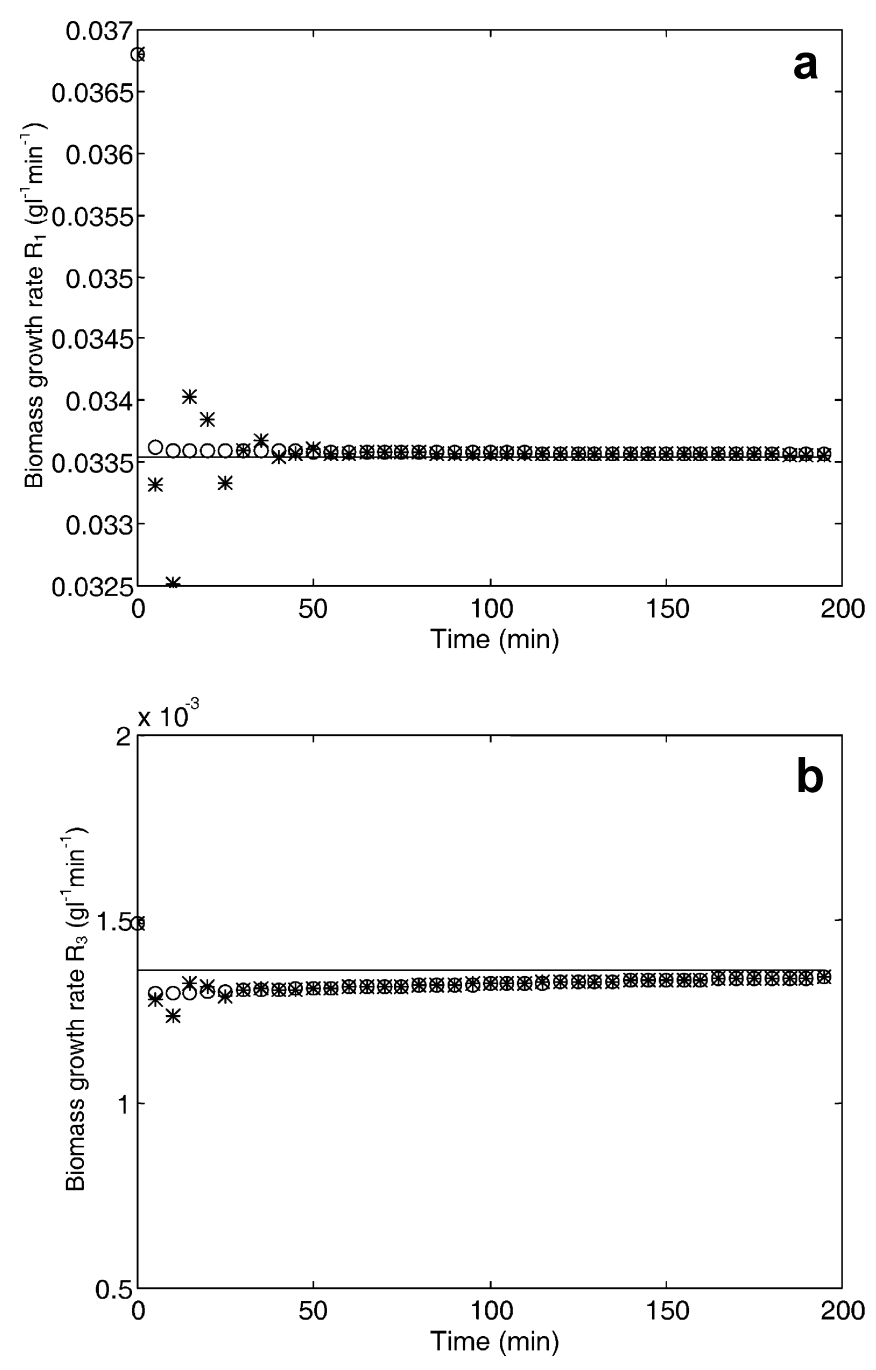

Fig. 1. Estimates of $R_{1}, R_{3}$, and the true values for the same parameters (lines) for continuous process with inexact initial conditions and different values of parameters $h_{1,2}: h_{1,2}=-1$ (open circles), $h_{1,2}=-0.1$ (asterisks), $C^{\mathrm{I}}=0.0001, C^{\mathrm{I}}=O, V_{1}(0)=3.5$

different values of $h_{1,2}{ }^{\text {III }}$. Comparison of the results shows that more exact $X$ estimates are obtained using algorithm II, while a better estimation of $R_{2}$ is obtained by algorithm III. This result can be explained by the fact, that the dynamical equations of $X$ and $R_{2}$ are not included in the structure of estimators III and II respectively, and the estimation of $X$ and $R_{2}$ is carried out indirectly.

For the batch-fed operation (step changes of dilution rate are given in Fig. 3), the initial values of variables and parameters $X, S_{1}, S_{2}, O, C, R_{1}, R_{2}, R_{3}$ are given in Table 2 . In Fig. 4, the simulation results of estimator I are plotted for different values of $h_{1,2}{ }^{\mathrm{I}}$. A good track of the changes in parameters $R_{1}$ and $R_{3}$ can be observed in Fig. $4 \mathrm{a}$ and $\mathrm{b}$ respectively. The results of estimator II are shown in Fig. 5. In Fig. $6, \hat{R}_{2}$ and $\hat{X}$ by estimator III are plotted for different values of $h_{1,2}$ III. A comparison of the results obtained by estimators II and III is given in Fig. 7. At the start of the operation ( $D=$ constant), more exact results are given by estimator II. The step changes in the dilution rate lead to changes in the values of the process state variables and parameters and to a more considerable deviation in $\hat{R}_{2}$ and $\hat{X}$ from their true values using estimator II. As shown on the figure, these deviations depend on the rates of change of $X$ and $R_{2}$. Towards the end of the operation, when the dilution rate is again constant, the errors of the estimates decrease.

On the basis of the results obtained, the following conclusions can be drawn:

1. Although the estimators do not have a small number of design parameters, the proposed tuning procedures reduce their number to three for estimator I: $C^{\mathrm{I}}, h_{1,2}$, and $V_{1}(0)$; four for estimator II: $h_{1,2}{ }^{\mathrm{II}}, C_{x 1}, C_{7}$ and $C_{x 2}$; and one for estimator III: $h_{1,2}$ III.

2 . For both kinds of process operation - continuous and batch-fed, better results are obtained with respect to $R_{1}$, in comparison with $R_{3}$.

3. Under a continuous process operation with inexact initial conditions, higher convergence of $\hat{X}$ is achieved by estimator II, while better estimation of $\hat{R}_{2}$ is carried out by estimator III.

4. Estimator III works better under dynamical conditions in comparison with estimator II, while in steady-state conditions without initial deviation of the estimates, estimator II gives better results.

5. In the simulations considered, the values of the tuning parameters are chosen using a trial-and-error approach. Other tuning methods may be proposed and applied for the experimental validation of these software sensors. For example, one possibility to be researched is a reasonable trade-off between noise sensitivity and convergence on the basis of criteria proposed in [26], using the experimental data.

\section{9}

\section{Conclusion}

An approach for estimating multiple biomass growth rates and biomass concentration for a class of aerobic bioprocesses is proposed. It is based on adaptive observer theory and requires on-line measurements of dissolved oxygen and carbon dioxide concentrations, as well as off-line measurements of biomass concentration. The approach includes two steps. In the first, an estimator of the biomass growth rates $R_{1}, R_{3}$, related to respiratory growth on substrate $S_{1}$ and respiratory growth on substrate $S_{2}(P)$ respectively is designed. In the second step, the biomass growth rate, $R_{2}$, related to the fermentative growth on substrate $S_{1}$, and biomass concentration $X$ are estimated, using two different estimators. One method is based on on-line measurements of dissolved oxygen concentration and off-line measurements of biomass concentrations, while the other needs only on-line measurements of carbon dioxide concentration.

The practical applicability of the proposed approach is a direct consequence of several important factors. The estimators (i) are not dependent on any particular models of the biomass growth rates, which are assumed to be unknown time-varying parameters, and (ii) require only on-line measurements of dissolved oxygen and carbon dioxide concentrations, which can be performed easily using reliable sensors. 

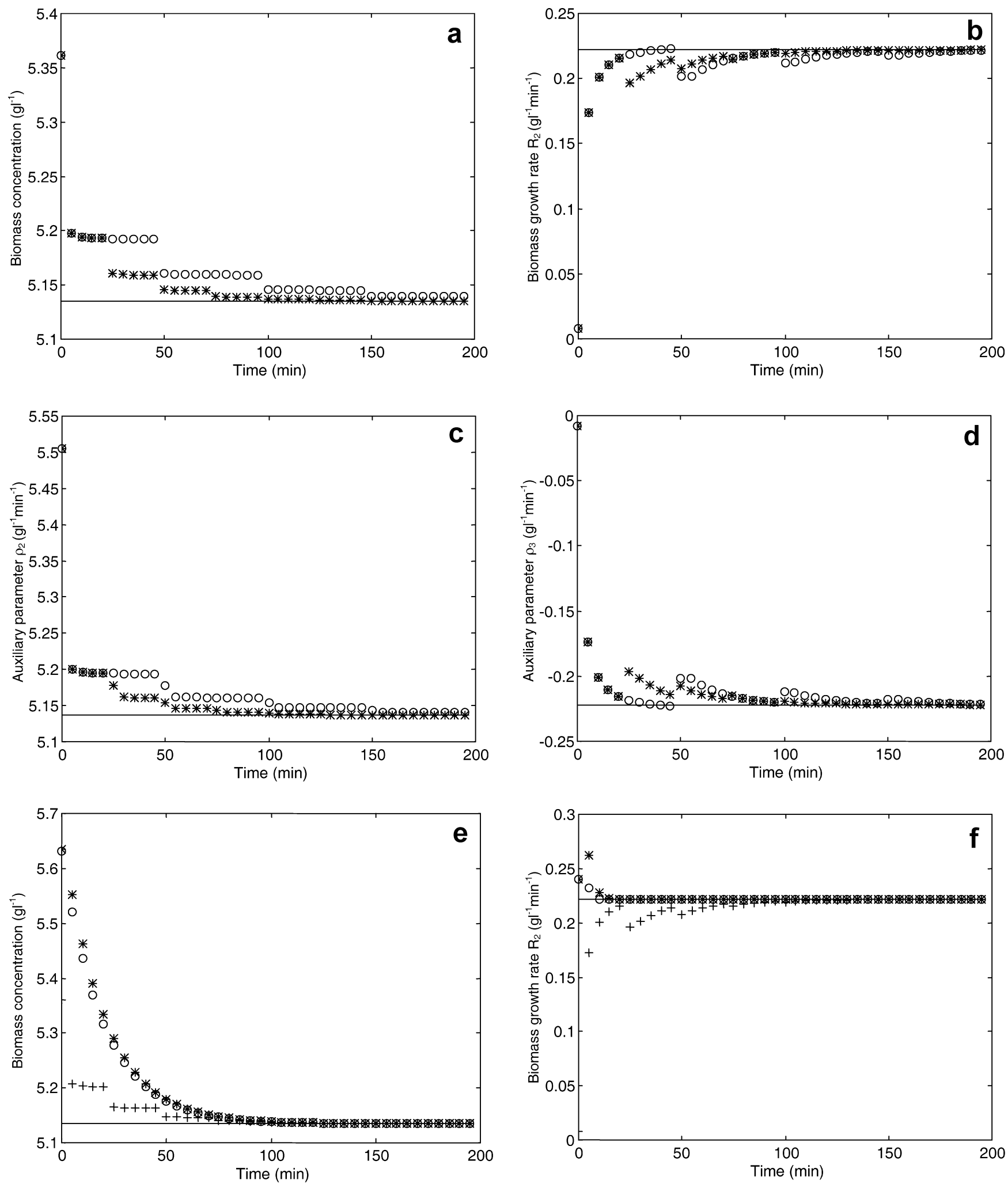

Fig. 2a-d. Estimates of $X, R_{2}, \varrho_{2}, \varrho_{3}$ and the true values (lines) for continuous process with inexact initial conditions and different sampling times $T_{x}: T_{x}=25 \mathrm{~min}$ (asterisks), $T_{x}=50 \mathrm{~min}$ (open circles). Values of design parameters: estimator I: $h_{1,2}{ }^{\mathrm{I}}=-1, C^{\mathrm{I}}=0.0001$, $C^{\mathrm{II}}=0, V_{1}(0)=3.5$; estimator II: $C^{\mathrm{II}}=0, C^{\mathrm{IV}}=1, h_{1,2}{ }^{\mathrm{II}}=-5$ and $C_{x 1}=C_{x 2}=C_{7}=5$. e, f Comparison of results by estimators II ( $p l u s$ signs) and by estimator III for different eigenvalues $h_{1,2}$ III: $h_{1,2}$ III $=-0.5$ (asterisks), $h_{1,2}$ III $=-1$ (open circles)

The results by simulation demonstrate the good performance of the proposed estimators for continuous and batch-fed conditions. It was shown that exact estimates could be obtained using different variants for tuning. The values of tuning parameters were chosen using a trial-and-error approach. The experimental validation of 


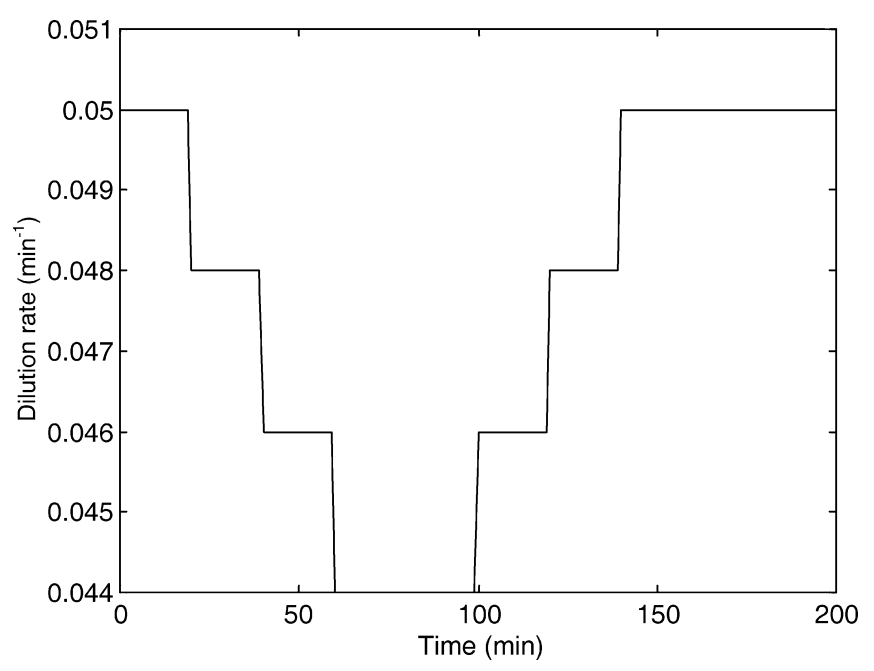

Fig. 3. Dilution rate for batch-fed process

the proposed estimation strategy raises the possibility of application to other tuning procedures, through the use of experimental data.

\section{Appendix}

\section{1}

\section{Proof of statement 1}

1. Defining $\tilde{R}_{1}^{*}=\tilde{R}_{1}-V_{1}\left(\tilde{\rho}_{1}\right)$, the following system is equivalent to the error system (15):

$$
\begin{aligned}
\frac{\mathrm{d}}{\mathrm{d} t}\left[\begin{array}{c}
\tilde{O} \\
\tilde{R}_{1}^{*} \\
\tilde{R}_{3} \\
\tilde{\rho}_{1}
\end{array}\right]=\left[\begin{array}{cccc}
-C_{1} & -k_{5} & -k_{6} & -V_{1} k_{5} \\
-C_{2} & -C^{I} & 0 & 0 \\
C_{3} & 0 & 0 & 0 \\
C_{4} & 0 & 0 & 0
\end{array}\right]\left[\begin{array}{c}
\tilde{O} \\
\tilde{R}_{1}^{*} \\
\tilde{R}_{3} \\
\tilde{\rho}_{1}
\end{array}\right] \\
+\left[\begin{array}{ll}
0 & 0 \\
0 & 0 \\
1 & 0 \\
0 & 1
\end{array}\right]\left[\begin{array}{c}
\frac{\mathrm{d} R_{3}}{\mathrm{~d} t} \\
\frac{\mathrm{d} \rho_{1}}{\mathrm{~d} t}
\end{array}\right]
\end{aligned}
$$

2. The homogeneous part of (36) can be written:

$$
\begin{aligned}
& \frac{\mathrm{d}}{\mathrm{d} t}\left[\begin{array}{c}
\tilde{O} \\
\tilde{R}_{1}^{*} \\
\tilde{R}_{3} \\
\tilde{\rho}_{1}
\end{array}\right]=\left[\begin{array}{cc}
\mathbf{B}_{11} & -\mathbf{B}_{21}^{\mathrm{T}} \\
\mathbf{B}_{21} \mathbf{P}_{1} & 0
\end{array}\right]\left[\begin{array}{c}
\tilde{O} \\
\tilde{R}_{1}^{*} \\
\tilde{R}_{3} \\
\tilde{\rho}_{1}
\end{array}\right] \text {, with } \\
& \mathbf{B}_{11}=\left[\begin{array}{ll}
-C_{1} & -k_{5} \\
-C_{2} & -C^{\mathrm{I}}
\end{array}\right] ; \mathbf{B}_{21}=\left[\begin{array}{cc}
k_{6} & 0 \\
k_{5} V_{1} & 0
\end{array}\right] ; \mathbf{P}_{1}=\left[\begin{array}{cc}
c_{1} & 0 \\
0 & 1
\end{array}\right]
\end{aligned}
$$

The matrix

$\mathbf{P}_{1} \mathbf{B}_{11}+\mathbf{B}_{11}^{\mathrm{T}} \mathbf{P}_{1}=\left[\begin{array}{cc}-2 C_{1} c_{1} & -k_{5} c_{1}-C_{2} \\ -k_{5} c_{1}-C_{2} & -2 C^{\mathrm{I}}\end{array}\right]$

is negative definite by assumptions A5-A8. Matrix $\mathbf{B}_{11}$ is stable by the same assumptions. $\mathbf{B}_{\mathbf{2 1}}$ is persistently exciting according to A9. Then, the exponential stability of (37)
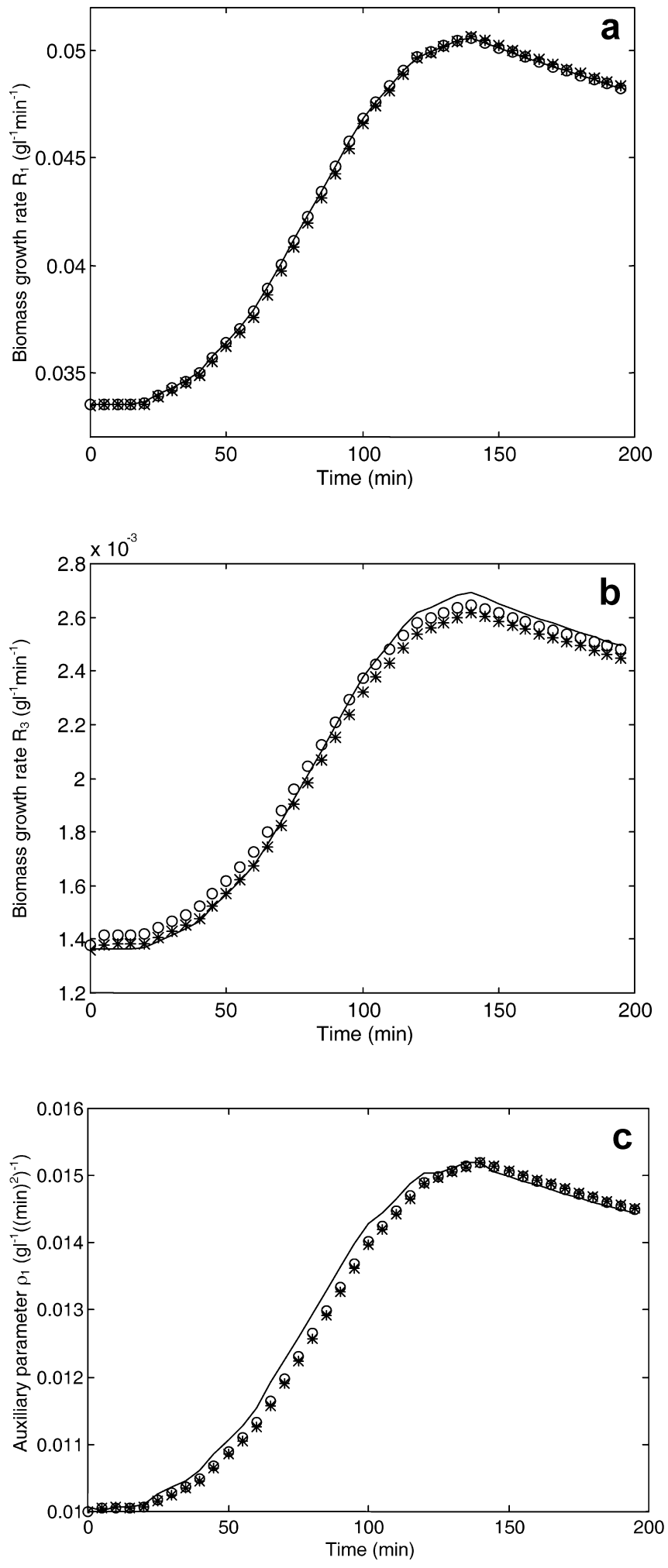

Fig. 4. Estimates of $R_{1}, R_{3}$ by estimator I and the true value of the same parameters (lines) for the batch-fed process for different values of the parameter $h_{1,2}: h_{1,2}=-5$ (open circles), $h_{1,2}=-0.5$ (asterisks), $C^{\mathrm{I}}=0.3, C^{\mathrm{I} 1}=1, V_{1}(0)=0.01$

follows from Theorem 3.2 [1].

3. The forcing term of (36) is bounded by assumptions A10 and A11.

4. Then it is a standard result of adaptive system theory that the state of system (15) is bounded (Theorem A2.6) [1]. 

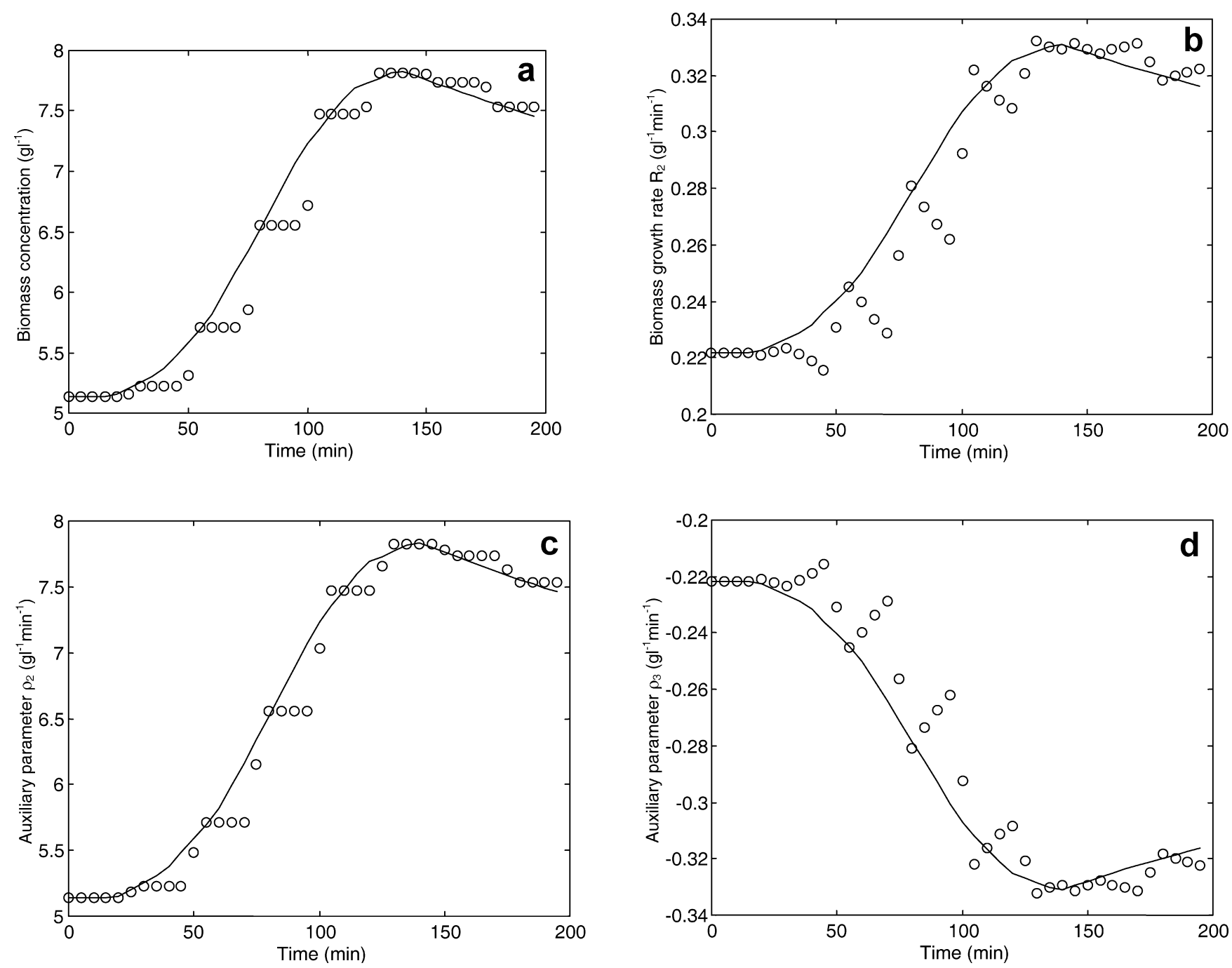

Fig. 5. Estimates of $X, R_{2}, \varrho_{2}, \varrho_{3}$ by estimator II and the true values (lines) for batch-fed process for sampling time $T_{x}=25$ min (open circles). Values of design parameters: estimator I: $h_{1,2}^{\mathrm{I}}=-5, C^{\mathrm{I}}=0.3, C^{\mathrm{I1}}=1, V_{1}(0)=0.01$, estimator II: $C^{\mathrm{III}}=0, C^{\mathrm{IV}}=1, h_{1,2}^{\mathrm{II}}=-5$ and $\mathrm{C}_{x 1=15}, \mathrm{C}_{x 2=} 0.1, \mathrm{C}_{7=} 10$

\section{2}

\section{Proof of statement 2}

Proof 1 . Defining $\tilde{X}^{*}=\tilde{X}+V_{2}\left(\tilde{\rho}_{3}\right)$, the following system is equivalent to the error system (26):

$\frac{\mathrm{d}}{\mathrm{d} t}\left[\begin{array}{c}\tilde{O} \\ \tilde{X}^{*} \\ \tilde{\rho}_{2} \\ \tilde{\rho}_{3}\end{array}\right]=\left[\begin{array}{cccc}-C_{5} & 1 & -1 & -V_{2} \\ -C_{6} & -C^{\mathrm{III}}-D & 0 & 0 \\ C_{7} & 0 & 0 & 0 \\ C_{8} & 0 & 0 & 0\end{array}\right]\left[\begin{array}{c}\tilde{O} \\ \tilde{X}^{*} \\ \tilde{\rho}_{2} \\ \tilde{\rho}_{3}\end{array}\right]$

$+\left[\begin{array}{ll}0 & 0 \\ 0 & 0 \\ 1 & 0 \\ 0 & 1\end{array}\right]\left[\begin{array}{c}\frac{\mathrm{d} \rho_{2}}{\mathrm{~d} t} \\ \frac{\mathrm{d} \rho_{3}}{\mathrm{~d} t}\end{array}\right]$
2. The homogeneous part of (38) can be written:

$$
\begin{aligned}
& \frac{\mathrm{d}}{\mathrm{d} t}\left[\begin{array}{c}
\tilde{O} \\
\tilde{X}^{*} \\
\tilde{\rho}_{2} \\
\tilde{\rho}_{3}
\end{array}\right]=\left[\begin{array}{cc}
\mathbf{B}_{21} & -\mathbf{B}_{22}^{\mathrm{T}} \\
\mathbf{B}_{22} \mathbf{P}_{2} & 0
\end{array}\right]\left[\begin{array}{c}
\tilde{O} \\
\tilde{X}^{*} \\
\tilde{\rho}_{2} \\
\tilde{\rho}_{3}
\end{array}\right] \text { with } \quad \mathbf{B}_{22}=\left[\begin{array}{cc}
1 & 0 \\
V_{2} & 0
\end{array}\right] \\
& \mathbf{P}_{2}=\left[\begin{array}{cc}
c_{2} & 0 \\
0 & 1
\end{array}\right]
\end{aligned}
$$

The matrix

$\mathbf{P}_{2} \mathbf{B}_{21}+\mathbf{B}_{21}^{\mathrm{T}} \mathbf{P}_{2}=\left[\begin{array}{cc}-2 C_{5} c_{2} & c_{2}-C_{6} \\ c_{2}-C_{6} & -2\left(C^{\mathrm{III}}+D\right)\end{array}\right]$

(38) is negative definite by assumptions A12-A15. Matrix $\mathbf{B}_{21}$ is stable by the same assumptions. $\mathbf{B}_{22}$ is persistently exciting according A16. Then, the exponential stability of (39) follows from Theorem 3.2 [1]. 
V. Lubenova et al.: Estimation of multiple biomass growth rates and biomass concentration in a class of bioprocesses
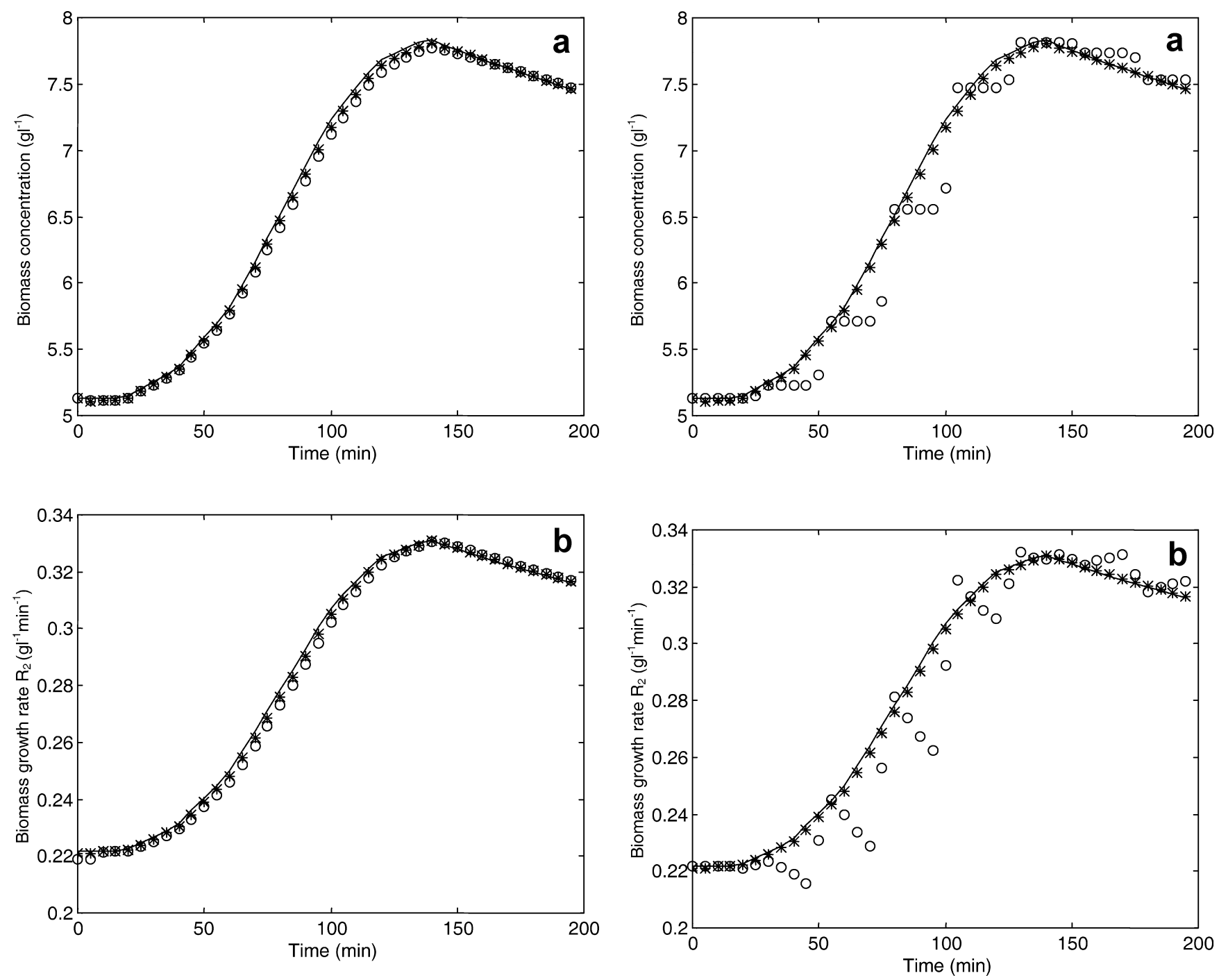

Fig. 6. Results by estimator III for batch-fed process for different eigenvalues $h_{1,2}$ III $h_{1,2}$ III $=-0.5$ (open circles), $h_{1,2}$ III $=-1$ (asterisks). Design parameters of estimator I are the same as in Fig. 5

Fig. 7. A comparison of results by estimators II (open circles) and by estimator III for $h_{1,2}{ }^{\text {III }}=-1$ (asterisks)

3. The forcing term of (38) is bounded by assumptions A17 and A18.

4. Then it is a standard result of adaptive system theory that the state of the system (26) is bounded (Theorem A2.6) [1].

\section{References}

1. Tatiraju S, Soroush M, Mutharasan R (1999) Biotechnol Bioeng 63:22-32

2. Bastin G, Dochain D (1990) On-line estimation and adaptive control of bioreactors. Elsevier Science, Amsterdam

3. Dochain D (1986) On-line parameter estimation, adaptive state estimation and adaptive control of fermentation processes. $\mathrm{PhD}$ Thesis, Université Catholique de Louvain, Belgium

4. Lubenova V (1993) State and parameter estimation of biotechnological processes. PhD Thesis, Technical University of Sofia

5. Cazzador L, Lubenova V (1995) Biotechnol Bioeng 47:626-632

6. Ferreira EC (1995) Identification and adaptive control of biotechnological processes. PhD Thesis, University of Porto, Portugal

7. Oliveira R, Ferreira EC, Oliveira F, Feyo de Azevedo S (1996) J Process Control 6:367-371

8. Lubenova V (1999) Bioprocess Eng 21:219-226

9. Perrier M, Feyo de Azevedo S, Ferreira EC, Dochain D (2000) Control Eng Practice 8:377-388

10. Pomerleau Y, Perrier P (1990) AIChE J 36:207-215

11. Farza M, Othman S, Hammouri H, Biston J (1997) Bioprocess Eng 17:143-150

12. Peres JP, Oliveira R, Feyo de Azevedo S (2001) Comput Chem Eng 25(4-6):783-791

13. Holmberg A, Ranta J (1982) Automatica 18:181-193

14. Shimizu H, Takamatsu T, Shioya S, and Suga KI (1989) Biotechnol Bioeng 33:354-364

15. Gudi RD, Shan SL, Gray MR (1994) Biotechnol Bioeng 44:1271

16. Gudi RD, Shan SL, Gray MR (1995) AIChE J 41:2451

17. Mutha RK, Cluett WR, Penlidis A (1997) AIChE J 43:3042

18. Halme A, Kuismin R, Korteniemi M (1986) A method to consider delayed laboratory analysis in state and parameter estimation of bioreactor. In: Johnson A (ed) Proceedings of the 1st IFAC Symposium on Modelling and Control of Biotechnological Processes. Pergamon Press, Oxford, pp 199-204

19. Petrova M, Georgieva O, Patarinska T (1995) Bioprocess Eng 12:103-107

20. Georgieva O, Patarinska T (in press) System analysis, modeling and simulation

21. Tatiraju S, Soroush M, Ogunnaike B (1999) AIChE J 45:769-780 
Bioprocess Biosyst Eng 25 (2003)

22. Sonnleitner B, Käpelli O (1986) Biotechnol Bioeng 28:927-937

23. Galvanauskas V, Simutis R, Volk N, Lübbert A (1998) Bioprocess Eng 18:227-234

24. Rocha I, Ferreira EC (2002) An integrated system for advanced monitoring and control of fed-batch fermentations of re- combinant E. coli. IFAC Computer Application in Biotechnology, 2001. Pergamon Press, Oxford, pp 349-354

25. Rocha I, Ferreira EC (2002) Anal Chim Acta 462:293-304

26. Claes J, Van Impe JF (1999) Bioprocess Eng 21:389-395 